\title{
NONIDENTIFIABILITY OF THE TWO-STATE MARKOVIAN ARRIVAL PROCESS
}

\author{
PEPA RAMÍREZ-COBO,${ }^{*}$ CNRS \\ ROSA E. LILLO $* * * * *$ AND \\ MICHAEL P. WIPER, ${ }^{* * * * * *}$ Universidad Carlos III de Madrid
}

\begin{abstract}
In this paper we consider the problem of identifiability for the two-state Markovian arrival process $\left(\mathrm{MAP}_{2}\right)$. In particular, we show that the $\mathrm{MAP}_{2}$ is not identifiable, providing the conditions under which two different sets of parameters induce identical stationary laws for the observable process.
\end{abstract}

Keywords: Markovian arrival process; Markov-modulated Poisson process; phase-type renewal process; identifiability problem

2010 Mathematics Subject Classification: Primary 60G55

Secondary $60 \mathrm{~J} 05$

\section{Introduction}

The Markovian arrival process (MAP) was defined in [16] and [17] as a generalization of the Poisson arrival process allowing for both dependence between arrivals and nonexponentially distributed interarrival times. The MAP is defined by two Markov processes: the first counts the number of arrivals and the second, an underlying Markov process, governs the state changes. At the end of a transition in a MAP an arrival may or may not occur and, although the transition holding times are exponentially distributed, the interarrival times do not, in general, follow an exponential distribution. Special cases of the MAP are phase-type renewal processes (which include both the Erlang and hyperexponential renewal processes) and nonrenewal processes such as the Markov-modulated Poisson process (MMPP). Stationary MAPs are dense in the family of all stationary point processes; see [1]. Another important property of MAPs is that the superposition of independent MAPs is again a MAP.

The MAP is a challenging process from both theoretical and applied points of view. From a theoretical perspective, the queueing system where the MAP governs the arrival process has been widely studied in the literature (see, for example, [15] and [19]). On the other hand, the MAP has been proposed in the literature as a suitable process for modeling teletraffic data; see, for example, [10], [11], [12], [18], and [25]. In this case, the MAP is used to fit data where only the interarrival times are observed and neither the underlying Markov chain nor the individual exponential holding times are available, and, thus, the observed arrival process is a hidden Markov process.

\footnotetext{
Received 13 July 2009; revision received 5 May 2010.

* Current address: Instituto Universitario de Matemáticas de la Universidad de Sevilla (IMUS), Facultad de Matemáticas, Universidad de Sevilla, Avda. Reina Mercedes, s/n, 41012 Sevilla, Spain. Email address: jrcobo@us.es

** Postal address: Departamento de Estadística, Universidad Carlos III. C/ Madrid, 126 - 28903 Getafe (Madrid), Spain.

*** Email address: lillo@est-econ.uc3m.es

**** Email address: mwiper@est-econ.uc3m.es
} 
When dealing with inference for hidden Markov processes, it is very common to encounter identifiability problems which imply that the likelihood function does not possess a unique maximum. Identifiability conditions for general hidden Markov processes are studied in [13], [14], and [21]. Several works have considered the identifiability issue with regards to some types of MAP. For example, identifiability of the MMPP and phase-type distributions was undertaken in [23], with results derived from a uniformization technique in combination with the findings of [13]. Unlike the MMPP, which can be identified (up to permutations of states), it is known that the phase-type distributions are not identifiable since, for example, any twostate phase-type distribution can be represented as a two-state Coxian distribution, which is not identifiable. Also, Green [6] and Bean and Green [3] investigated when a MAP is a Poisson process. He and Zhang [7], [8], [9] used the so-called spectral polynomial algorithm to construct Coxian representations for phase-type distributions whose generators have only real eigenvalues. Finally, a new parameterization which provides an identifiable Coxian model can be found in [2]. In this paper we address the problem of identifiability of the general two-state MAP, or $\mathrm{MAP}_{2}$. We prove the lack of identifiability of the $\mathrm{MAP}_{2}$ using an approach that allows us to construct an equivalent $\mathrm{MAP}_{2}$ for any given $\mathrm{MAP}_{2}$.

The paper is organized as follows. In Section 2 we introduce the MAP and its main properties. In Section 3 we study when two $\mathrm{MAP}_{2}$ s have the same interarrival time distributions, a necessary condition for nonidentifiability. We call this property weak equivalence. In Section 4 we consider the joint distribution of a sequence of interarrival times generated from the $\mathrm{MAP}_{2}$ and show that there are at least two different parameterizations of the $\mathrm{MAP}_{2}$ giving rise to the same joint distribution, thus proving the nonidentifiability of the $\mathrm{MAP}_{2}$. Finally, in Section 5 we provide conclusions and some possible extensions of this work.

\section{The MAP and its main properties}

Consider an irreducible, continuous, Markov process $J(t)$ with state space $\delta=\{1, \ldots, m\}$ and generator matrix $\boldsymbol{D}$. Let $N(t)$ represent the cumulative number of arrivals in $(0, t]$. A MAP, represented by $\{J(t), N(t)\}$, behaves as follows: the initial state $i_{0} \in \delta$ is generated according to an initial probability vector $\boldsymbol{\theta}=\left(\theta_{1}, \ldots, \theta_{m}\right)$ and at the end of an exponentially distributed sojourn time in state $i$, with mean $1 / \lambda_{i}$, two possible state transitions can occur. Firstly, with probability $0 \leq p_{i j 1} \leq 1$, a single arrival occurs and the MAP enters a state $j \in \delta$, which may be the same as $(j=i)$ or different from $(j \neq i)$ the previous state. Secondly, with probability $0 \leq p_{i j 0} \leq 1$, no arrival occurs and the MAP enters a different state, $j \neq i$. Given that from all states a transition must occur to a different state without an arrival or to any state with an arrival, then, for $1 \leq i \leq m$,

$$
\sum_{j=1, j \neq i}^{m} p_{i j 0}+\sum_{j=1}^{m} p_{i j 1}=1 .
$$

When $m=2$, we have a two-state MAP, denoted by MAP 2 . Figure 1 illustrates the different transitions that can occur in this process by means of a transition diagram.

Define the matrices $\boldsymbol{P}_{0}=\left(p_{i j 0}\right)_{i, j \in \delta}$ and $\boldsymbol{P}_{1}=\left(p_{i j 1}\right)_{i, j \in \delta}$, where $p_{i i 0}=0$. Then the MAP is defined by the set $\left\{\boldsymbol{\theta}, \boldsymbol{\lambda}, \boldsymbol{P}_{0}, \boldsymbol{P}_{1}\right\}$, where $\boldsymbol{\lambda}=\left(\lambda_{1}, \ldots, \lambda_{m}\right)$. Alternatively, the MAP can be characterized by the rate matrices, $\boldsymbol{D}_{0}=\left(d_{i j 0}\right)_{i, j \in \delta}$ and $\boldsymbol{D}_{1}=\left(d_{i j 1}\right)_{i, j \in S}$, where $d_{i i 0}=-\lambda_{i}$, $d_{i j 0}=\lambda_{i} p_{i j 0}$ for $j \neq i$, and $d_{i j 1}=\lambda_{i} p_{i j 1}$ for $1 \leq i, j \leq m$. This definition implies that $\boldsymbol{D} \equiv \boldsymbol{D}_{0}+\boldsymbol{D}_{1}$ is the infinitesimal generator of the underlying Markov process. Intuitively, $\boldsymbol{D}_{0}$ can be thought of as governing transitions that do not generate arrivals and $\boldsymbol{D}_{1}$ can be thought 


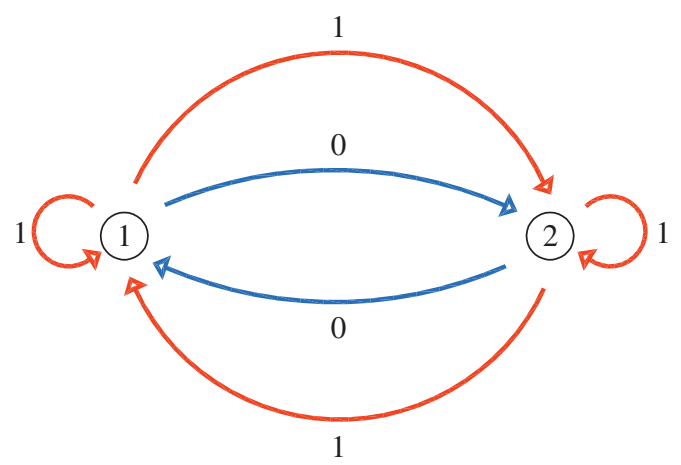

FIGURE 1: Transition diagram for the $\mathrm{MAP}_{2}$. Here 0 and 1 illustrate moves without and with arrivals, respectively.

of as governing transitions that do generate arrivals. The stationary probability vector of the Markov process with generator $\boldsymbol{D}$ is $\pi=\left(\pi_{1}, \ldots, \pi_{m}\right)$, which satisfies

$$
\pi D=\mathbf{0}, \quad \pi e=1,
$$

where $\boldsymbol{e}$ is an $m \times 1$ column vector of $1 \mathrm{~s}$. Thus, $\pi_{j}$ represents the stationary probability that the process is in state $j$, for $j=1, \ldots, m$. The MMPP can be defined as a simplified MAP where the matrix $\boldsymbol{D}_{1}$ (and thus, $\boldsymbol{P}_{1}$ ) is diagonal (see [15]). This implies that arrivals can only occur in transitions to the same state.

Some important properties of the MAP are as follows. Firstly, it is known (see [4]) that the MAP can be regarded as a Markov renewal process. Let $X_{n}$ be the state of the MAP at the time of the $n$th arrival, and let $T_{n}$ be the time between the $(n-1)$ th and $n$th arrival. Then $\left\{X_{n-1}, T_{n}\right\}_{n=1}^{\infty}$ is a Markov renewal process with semi-Markovian kernel given by

$$
\int_{0}^{t} \mathrm{e}^{\boldsymbol{D}_{0} t} \boldsymbol{D}_{1} \mathrm{~d} t=\left(\boldsymbol{I}-\mathrm{e}^{\boldsymbol{D}_{0} t}\right)\left(-\boldsymbol{D}_{0}\right)^{-1} \boldsymbol{D}_{1} .
$$

Therefore, $\left\{X_{n}\right\}_{n=1}^{\infty}$ is a Markov chain, where from (2.1) the transition matrix can be computed as

$$
\boldsymbol{P}^{\star}=\left(\boldsymbol{I}-\boldsymbol{P}_{0}\right)^{-1} \boldsymbol{P}_{1}
$$

Also, it can be shown that the stationary distribution, $\phi$, is given by

$$
\phi=\left(\pi D_{1} e\right)^{-1} \pi D_{1}
$$

See Appendix A for the proof.

Secondly, let the random variable $T_{1}$ denote the time to the first arrival in a MAP. Then, from [4], the cumulative distribution function $(\mathrm{CDF})$ of $T_{1}$ is given by

$$
F_{T_{1}}(t)=\boldsymbol{\theta}\left(\boldsymbol{I}-\mathrm{e}^{\boldsymbol{D}_{0} t}\right)\left(-\boldsymbol{D}_{0}\right)^{-1} \boldsymbol{D}_{1} \boldsymbol{e} \text { for } t \geq 0 .
$$

If $T$ represents the stationary interarrival time distribution, it can be shown that

$$
F_{T}(t)=\boldsymbol{\phi}\left(\boldsymbol{I}-\mathrm{e}^{\boldsymbol{D}_{0} t}\right)\left(-\boldsymbol{D}_{0}\right)^{-1} \boldsymbol{D}_{1} \boldsymbol{e} \quad \text { for } t \geq 0 .
$$


Finally, the Laplace-Stieltjes transform of the first $n$ interarrival times of a stationary MAP is given by

$$
f_{T ; \boldsymbol{D}_{0}, \boldsymbol{D}_{1}}^{*}\left(s_{1}, \ldots, s_{n}\right)=\boldsymbol{\phi}\left(s_{1} \boldsymbol{I}-\boldsymbol{D}_{0}\right)^{-1} \boldsymbol{D}_{1} \cdots\left(s_{n} \boldsymbol{I}-\boldsymbol{D}_{0}\right)^{-1} \boldsymbol{D}_{1} \boldsymbol{e},
$$

or, equivalently,

$$
f_{T ; \boldsymbol{D}_{0}, \boldsymbol{D}_{1}}^{*}\left(s_{1}, \ldots, s_{n}\right)=\boldsymbol{\phi} \prod_{i=1}^{n} \boldsymbol{\Delta}\left(s_{i}\right) \boldsymbol{e}
$$

where

$$
\boldsymbol{\Delta}(s)=\left(s \boldsymbol{I}-\boldsymbol{D}_{0}\right)^{-1} \boldsymbol{D}_{1} .
$$

For a more detailed description and further properties of the MAP (and batch MAP (BMAP)), see, for example, [4] or [15].

\section{Weak equivalence}

There have been a number of examples of fitting MMPPs to Internet data traces. In most applications, the two-state case has been considered (see, for example, [5], [22], or [24]). The MMPPs, despite being simplified MAPs (the matrix $\boldsymbol{P}_{1}$ is diagonal, and, thus, they are characterized by two fewer parameters), are complex processes and usually two states, at most three, are enough to capture the data behavior.

From now on we consider the two-state MAP, or $\mathrm{MAP}_{2}$, characterized by $\mathcal{M} \equiv\left\{\boldsymbol{\theta}, \boldsymbol{D}_{0}, \boldsymbol{D}_{1}\right\}$, where

$$
\boldsymbol{\theta}=(\theta, 1-\theta), \quad \boldsymbol{D}_{0}=\left(\begin{array}{cc}
x & y \\
z & u
\end{array}\right), \quad \boldsymbol{D}_{1}=\left(\begin{array}{cc}
w & -x-y-w \\
v & -z-u-v
\end{array}\right)
$$

and

$$
\begin{array}{lrl}
x=-\lambda_{1}, & y=\lambda_{1} p_{120}, & w=\lambda_{1} p_{111}, \\
z=\lambda_{2} p_{210}, & u=-\lambda_{2}, & v=\lambda_{2} p_{211} .
\end{array}
$$

The stationary probability distribution is $\phi=(\phi, 1-\phi)$, where

$$
\phi=\frac{w z-v x}{w z-v x-z y-v y+x u+w u} .
$$

When modeling real data, usually only the times between arrivals are observed, and, thus, the interest when making an inference for the MAP is focused on the embedded Markov renewal process $\left\{X_{n-1}, T_{n}\right\}_{n=1}^{\infty}$. As a preliminary step to studying the identifiability of the $\mathrm{MAP}_{2}$, we study the conditions under which two $\mathrm{MAP}_{2}$ s possess the same marginal interarrival time distributions. For two such $\mathrm{MAP}_{2} \mathrm{~s}$, we will say that they are weakly equivalent.

Definition 3.1. Let $\mathcal{M}$ represent a $\mathrm{MAP}_{2}$ with parameters $\left\{\boldsymbol{\theta}, \boldsymbol{D}_{0}, \boldsymbol{D}_{1}\right\}$ as in (3.1). Then we say that another $\mathrm{MAP}_{2}, \tilde{\mathcal{M}} \equiv\left\{\tilde{\boldsymbol{\theta}}, \tilde{\boldsymbol{D}}_{0}, \tilde{\boldsymbol{D}}_{1}\right\}$ is weakly equivalent to $\mathcal{M}$ if and only if

$$
T_{n} \stackrel{\mathrm{D}}{=} \tilde{T}_{n} \quad \text { for all } n \geq 1,
$$

where $T_{n}$ and $\tilde{T}_{n}$ represent the times between the $(n-1)$ th and $n$th arrivals under both models, and ' $\stackrel{\text { D }}{=}$ denotes equality in distribution. 
The term weak is employed because equivalence is expressed in a marginal sense. Since the interarrival times in a $\mathrm{MAP}_{2}$ are not independent, condition (3.3) is necessary but insufficient for nonidentifiability. A general condition looking at the joint density of the sequence of interarrival times is analyzed in Section 4.

Given a known $\mathrm{MAP}_{2}, \mathcal{M}$ as in (3.1), define the constant

$$
c=z+u-x-y .
$$

Note that if $c=0$ then the rate until an arrival occurs from state 1 coincides with that of state 2 . This implies that the observable process (that is, where arrivals occur) behaves like a Poisson process, with a single arrival rate. Thus, we will assume that $c \neq 0$. In addition, define the matrix $\boldsymbol{\Phi}$ as that whose rows are composed of the stationary vector $\boldsymbol{\phi}$. Suppose that $\boldsymbol{P}^{\star}=\boldsymbol{\Phi}$. Then it is immediately clear that there are many weakly equivalent $\mathrm{MAP}_{2} \mathrm{~s}$, for example, any $\tilde{\mathcal{M}}=\left\{\tilde{\boldsymbol{\theta}}, \boldsymbol{D}_{0}, \boldsymbol{D}_{1}\right\}$ is equivalent to $\mathcal{M}=\left\{\boldsymbol{\theta}, \boldsymbol{D}_{0}, \boldsymbol{D}_{1}\right\}$. We can thus also assume that $\boldsymbol{P}^{\star} \neq \boldsymbol{\Phi}$.

Theorem 3.1 gives the conditions for $\tilde{\mathcal{M}}$ to be weakly equivalent to $\mathcal{M}$.

Theorem 3.1. Let $\mathcal{M}$ be a $M A P_{2}$ as in (3.1) with stationary distribution $\boldsymbol{\phi}$, and let $\tilde{\mathcal{M}}$ be another $M A P_{2}$ with stationary distribution $\tilde{\boldsymbol{\phi}}$. Let $T$ and $\tilde{T}$ represent the interarrival times in the stationary versions of $\mathcal{M}$ and $\tilde{\mathcal{M}}$. Assume that

(A1) $c \neq 0$ and $\tilde{c} \neq 0$;

(A2) $\boldsymbol{P}^{\star} \neq \boldsymbol{\Phi}$ or $\tilde{\boldsymbol{P}}^{\star} \neq \tilde{\boldsymbol{\Phi}}$.

Then, $\tilde{\mathcal{M}}$ is weakly equivalent to $\mathcal{M}$ if and only if

(C1) $T \stackrel{\mathrm{D}}{=} \tilde{T}$; and

(C2) $(\boldsymbol{\theta}, \tilde{\boldsymbol{\theta}})=(\boldsymbol{\phi}, \tilde{\boldsymbol{\phi}})$.

As will be shown in the proof of Theorem 3.1 (see Appendix B), (C1) is equivalent to

$$
\frac{\alpha s+\gamma}{s^{2}+\beta s+\gamma}=\frac{\tilde{\alpha} s+\tilde{\gamma}}{s^{2}+\tilde{\beta} s+\tilde{\gamma}} \text { for all } s,
$$

where the coefficients $\alpha, \beta$, and $\gamma$ are given by

$$
\alpha=\theta(z+u-x-y)-(z+u), \quad \beta=-x-u, \quad \gamma=x u-y z
$$

similarly define $\tilde{\alpha}, \tilde{\beta}$, and $\tilde{\gamma}$.

Theorem 3.1 thus provides a straightforward way to check if two given $\mathrm{MAP}_{2} \mathrm{~s}$ share the same interarrival distribution.

Example 3.1. As an example, let us consider the $\mathrm{MAP}_{2}$ defined by

$$
\boldsymbol{D}_{0}=\left(\begin{array}{cc}
-20 & 6 \\
0.15 & -0.5
\end{array}\right), \quad \boldsymbol{D}_{1}=\left(\begin{array}{cc}
12.228 & 1.772 \\
0.0426 & 0.3074
\end{array}\right)
$$

or, alternatively,

$$
\boldsymbol{P}_{0}=\left(\begin{array}{cc}
0 & 0.3 \\
0.3 & 0
\end{array}\right), \quad \boldsymbol{P}_{1}=\left(\begin{array}{cc}
0.6114 & 0.0886 \\
0.0852 & 0.6148
\end{array}\right)
$$


and exponential rates $\left(\lambda_{1}, \lambda_{2}\right)=(20,0.5)$. Suppose that the initial probability vector is equal to the stationary distribution, $\boldsymbol{\theta}=\boldsymbol{\phi}=(0.496,0.504)$. The transition probability matrix, $\boldsymbol{P}^{\star}$, is computed from (2.2):

$$
\boldsymbol{P}^{\star}=\left(\begin{array}{cc}
0.7 & 0.3 \\
0.2952 & 0.7048
\end{array}\right) \neq \boldsymbol{\Phi} .
$$

Consider another $\mathrm{MAP}_{2}$ with parameters

$$
\tilde{\boldsymbol{D}}_{0}=\left(\begin{array}{cc}
-19.7 & 10.835 \\
0.6146 & -0.8
\end{array}\right), \quad \tilde{\boldsymbol{D}}_{1}=\left(\begin{array}{cc}
7.1452 & 1.7198 \\
0.1443 & 0.0411
\end{array}\right),
$$

or, alternatively,

$$
\tilde{\boldsymbol{P}}_{0}=\left(\begin{array}{cc}
0 & 0.5500 \\
0.7682 & 0
\end{array}\right), \quad \tilde{\boldsymbol{P}}_{1}=\left(\begin{array}{ll}
0.3627 & 0.0873 \\
0.1804 & 0.0514
\end{array}\right),
$$

and exponential rates $\left(\tilde{\lambda}_{1}, \tilde{\lambda}_{2}\right)=(19.7,0.8)$. Assume that $\tilde{\boldsymbol{\theta}}=\tilde{\boldsymbol{\phi}}=(0.799,0.201)$. The transition probability matrix, $\tilde{\boldsymbol{P}}^{\star}$, is

$$
\tilde{\boldsymbol{P}}^{\star}=\left(\begin{array}{cc}
0.8 & 0.2 \\
0.79 & 0.21
\end{array}\right) \neq \boldsymbol{\Phi}
$$

It can be seen that $c=13.65 \neq 0$ and $\tilde{c}=-8.6796 \neq 0$, and that (3.5) holds. Therefore, from Theorem 3.1, the processes are weakly equivalent, as shown in Figure 2, which depicts the CDF of the time between two arrivals in the stationary version for both $\mathrm{MAP}_{2} \mathrm{~s}$.

More results similar to Theorem 3.1, when assumptions (A1) and (A2) are relaxed (and, thus, the number of weakly equivalent $\mathrm{MAP}_{2} \mathrm{~s}$ to a fixed $\mathrm{MAP}_{2}$ increases), and extensions to the three-state case MAP can be found in [20].

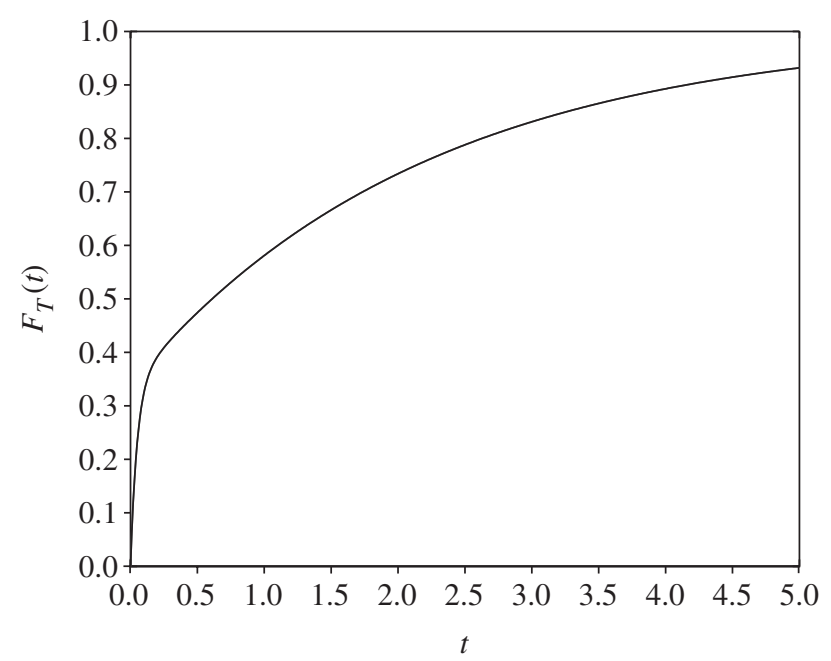

Figure 2: CDF of $T$, the time until the next arrival in the stationary version, in Example 1. As $\boldsymbol{\theta}=\boldsymbol{\phi}$, then $T \stackrel{\text { D }}{=} T_{1}$ (similarly, $\tilde{T} \stackrel{\text { D }}{=} \tilde{T}_{1}$ ), and, thus, $T \stackrel{\text { D }}{=} T_{1} \stackrel{\text { D }}{=} \tilde{T} \stackrel{\text { D }}{=} \tilde{T}_{1}$. 


\section{Nonidentifiability of the $\mathrm{MAP}_{2}$}

In this section we prove that the $\mathrm{MAP}_{2}$ is a nonidentifiable process. Following Theorem 3.1, all $\mathrm{MAP}_{2} \mathrm{~s}$ will be assumed to be stationary, and $\mathcal{M}$ and $\tilde{\mathcal{M}}$ will denote the sets $\left\{\boldsymbol{\phi}, \boldsymbol{D}_{0}, \boldsymbol{D}_{1}\right\}$ and $\left\{\tilde{\boldsymbol{\phi}}, \tilde{\boldsymbol{D}}_{0}, \tilde{\boldsymbol{D}}_{1}\right\}$, respectively, from now on. Our definition of nonidentifiability follows [23].

Definition 4.1. The $\mathrm{MAP}_{2}$ is a nonidentifiable process if, for any fixed $\mathrm{MAP}_{2}, \mathcal{M}$, there exists another $\mathrm{MAP}_{2}, \tilde{\mathcal{M}}$, such that

$$
\left(T_{1}, \ldots, T_{n}\right) \stackrel{\mathrm{D}}{=}\left(\tilde{T}_{1}, \ldots, \tilde{T}_{n}\right) \quad \text { for all } n \geq 1 .
$$

Note that condition (4.1) is equivalent to the equality of the Laplace transforms,

$$
f_{T ; \boldsymbol{D}_{0}, \boldsymbol{D}_{1}}^{*}\left(s_{1}, \ldots, s_{n}\right)=f_{\tilde{T} ; \tilde{\boldsymbol{D}}_{0}, \tilde{\boldsymbol{D}}_{1}}^{*}\left(s_{1}, \ldots, s_{n}\right)
$$

for all $n, s$. We will show that, given a $\mathrm{MAP}_{2}, \mathcal{M}$, as in (3.1), there always exists a differently parameterized $\tilde{\mathcal{M}}$ such that (4.2) holds for all $n, s$. Indeed, we will prove that if (4.2) holds for $n=1,2$ then it will hold for all $n$.

Let us first consider the following result that gives the conditions under which (4.2) holds for $n=1,2$.

Proposition 4.1. Let $\mathcal{M}$ and $\tilde{\mathcal{M}}$ be two $M A P_{2}$ s. Let $\alpha, \beta, \gamma, \delta_{1}, \delta_{2}$ be defined as

$$
\begin{aligned}
\alpha= & \phi(z+u-x-y)-(z+u), \\
\beta= & -x-u, \\
\gamma= & x u-y z, \\
\delta_{1}= & \phi\left((z+u-x-y)(w-v)+(x+y)(z+u)-(z+u)^{2}\right)+(z+u-x-y) v \\
& +(z+u)^{2}, \\
\delta_{2}= & \phi(x+y-z-u)(u w-y v-x v+z w)+(x+y-z-u)(x v-z w)-(u+z) \gamma ;
\end{aligned}
$$

similarly define $\tilde{\alpha}, \tilde{\beta}, \tilde{\gamma}, \tilde{\delta}_{1}$, and $\tilde{\delta}_{2}$. Then, if

$$
\tilde{\alpha}=\alpha, \quad \tilde{\beta}=\beta, \quad \tilde{\gamma}=\gamma, \quad \tilde{\delta}_{1}=\delta_{1}, \quad \tilde{\delta}_{2}=\delta_{2},
$$

the equality of Laplace transforms, (4.2), holds for all $s$ and $n=1,2$.

Proof. See Appendix C.

The following result gives the solutions to (4.3).

Proposition 4.2. Consider a MAP $P_{2}$ as in (3.1). For all $\tilde{u}<0$ and all $\tilde{z}>0$, let $\tilde{x}(\tilde{u}, \tilde{z}), \tilde{y}(\tilde{u}, \tilde{z})$, $\tilde{v}(\tilde{u}, \tilde{z})$, and $\tilde{w}(\tilde{u}, \tilde{z})$ be defined as

$$
\begin{aligned}
\tilde{x}(\tilde{u}, \tilde{z})= & -\tilde{u}+x+u, \\
\tilde{y}(\tilde{u}, \tilde{z})= & -\frac{1}{\tilde{z}}\left(\tilde{u}^{2}-\tilde{u} x-\tilde{u} u+x u-z y\right), \\
\tilde{v}(\tilde{u}, \tilde{z})= & \frac{-\tilde{z}(v x+v y-w z-w u+w \tilde{z}-z \tilde{z}-z \tilde{u})}{-\tilde{u} u-u \tilde{z}+x u+\tilde{u}^{2}+2 \tilde{u} \tilde{z}+\tilde{z}^{2}-\tilde{u} x-\tilde{z} x-z y} \\
& +\frac{-\tilde{z}\left(-\tilde{u} u+\tilde{z}^{2}+2 \tilde{u} \tilde{z}-u \tilde{z}-v \tilde{z}-\tilde{u} v+w \tilde{u}+\tilde{u}^{2}\right)}{-\tilde{u} u-u \tilde{z}+x u+\tilde{u}^{2}+2 \tilde{u} \tilde{z}+\tilde{z}^{2}-\tilde{u} x-\tilde{z} x-z y},
\end{aligned}
$$




$$
\begin{aligned}
\tilde{w}(\tilde{u}, \tilde{z})= & \frac{z \tilde{u} x+2 \tilde{z} x u-\tilde{z} z y-x u^{2}+z x \tilde{z}+z u \tilde{z}+\tilde{u} u^{2}+u z y}{-\tilde{u} u-u \tilde{z}+x u+\tilde{u}^{2}+2 \tilde{u} \tilde{z}+\tilde{z}^{2}-\tilde{u} x-\tilde{z} x-z y} \\
& +\frac{-z x u-2 \tilde{z} \tilde{u} x+z^{2} y+u \tilde{z} v-w z y+z v y-\tilde{z}^{2} u-3 \tilde{u} \tilde{z} u-\tilde{u} \tilde{z} v}{-\tilde{u} u-u \tilde{z}+x u+\tilde{u}^{2}+2 \tilde{u} \tilde{z}+\tilde{z}^{2}-\tilde{u} x-\tilde{z} x-z y} \\
& +\frac{v \tilde{u} x-\tilde{z} v y+2 \tilde{u} x u+v \tilde{u} u-w \tilde{u} x-w u \tilde{u}+2 \tilde{u}^{2} \tilde{z}+z \tilde{u} u+w u x}{-\tilde{u} u-u \tilde{z}+x u+\tilde{u}^{2}+2 \tilde{u} \tilde{z}+\tilde{z}^{2}-\tilde{u} x-\tilde{z} x-z y} \\
& +\frac{\tilde{z}^{2} \tilde{u}-v \tilde{u}^{2}-\tilde{u}^{2} x+\tilde{z} w z+\tilde{u} \tilde{z} w-\tilde{u} \tilde{z} z-v x u+w \tilde{u}^{2}-z \tilde{u}^{2}+\tilde{u}^{3}}{-\tilde{u} u-u \tilde{z}+x u+\tilde{u}^{2}+2 \tilde{u} \tilde{z}+\tilde{z}^{2}-\tilde{u} x-\tilde{z} x-z y} \\
& +\frac{-z y \tilde{u}+\tilde{z} u^{2}-\tilde{z}^{2} x-2 \tilde{u}^{2} u-\tilde{z} w x}{-\tilde{u} u-u \tilde{z}+x u+\tilde{u}^{2}+2 \tilde{u} \tilde{z}+\tilde{z}^{2}-\tilde{u} x-\tilde{z} x-z y} .
\end{aligned}
$$

Then, the set $\{\tilde{u}, \tilde{z}, \tilde{x}(\tilde{u}, \tilde{z}), \tilde{y}(\tilde{u}, \tilde{z}), \tilde{v}(\tilde{u}, \tilde{z}), \tilde{w}(\tilde{u}, \tilde{z})\}$ solves the system of equations given by (4.3).

The proof of Proposition 4.2 is tedious but straightforward, involving solving the system of equations (4.3) by conventional methods, and substituting $\phi$ and $\tilde{\phi}$ from their definitions (see (3.2)). Although Proposition 4.2 gives an infinite number of solutions to the system of equations (4.3), a priori, the values of $\tilde{x}, \tilde{y}, \tilde{v}$, and $\tilde{w}$ may not define a $\mathrm{MAP}_{2}$. The following theorem details how to select feasible values of $\tilde{u}$ and $\tilde{z}$ in the sense that $\tilde{x}<0, \tilde{y}>0, \tilde{v}>0$, $\tilde{w}>0,-\tilde{x}-\tilde{y}-\tilde{w}>0,-\tilde{z}-\tilde{u}-\tilde{v}>0$, and $\tilde{\phi} \in[0,1]$, that is, it provides a way to choose $\tilde{u}$ and $\tilde{z}$ so that $\tilde{\mathcal{M}}$ is equivalent to $\mathcal{M}$.

Theorem 4.1. Consider a $M A P_{2}, \mathcal{M}$, as in (3.1), and define

$$
\begin{aligned}
& \varepsilon_{1}=-x \\
& \varepsilon_{2}=\frac{u-x}{2}, \\
& \varepsilon_{3}=\frac{z(1-\phi)}{\phi}, \\
& \varepsilon_{4}=\frac{(u-x)+\sqrt{(x-u)^{2}+4 z y}}{2}, \\
& \varepsilon_{5}=-\frac{z}{v}(z+u+v), \\
& \varepsilon_{6}=-\frac{z}{2 v}\left[(u+v+z+w)-\sqrt{(u+v+z+w)^{2}+4 v(-w-y-x)}\right] .
\end{aligned}
$$

Let $\varepsilon$ be chosen from

$$
\begin{aligned}
& 0<\varepsilon<\min \left\{\varepsilon_{1}, \varepsilon_{2}, \varepsilon_{3}, \varepsilon_{4}, \varepsilon_{5}, \varepsilon_{6}\right\} \quad \text { if } x<u, \\
& 0<\varepsilon<\min \left\{\varepsilon_{1}, \varepsilon_{3}, \varepsilon_{4}, \varepsilon_{5}, \varepsilon_{6}\right\} \quad \text { if } x=u,
\end{aligned}
$$

and set $\tilde{u} \equiv u-\varepsilon$ and $\tilde{z} \equiv z+\varepsilon$. Then there exist an infinite number of $M A P_{2} s, \tilde{\mathcal{M}}$, given by $\mathcal{F}=\{\tilde{u}, \tilde{z}, \tilde{x}(\tilde{u}, \tilde{z}), \tilde{y}(\tilde{u}, \tilde{z}), \tilde{v}(\tilde{u}, \tilde{z}), \tilde{w}(\tilde{u}, \tilde{z})\}$, where $\tilde{x}(\tilde{u}, \tilde{z}), \tilde{y}(\tilde{u}, \tilde{z}), \tilde{v}(\tilde{u}, \tilde{z})$, and $\tilde{w}(\tilde{u}, \tilde{z})$ are defined by (4.4)-(4.7), such that (4.3) holds.

Note that the case $x>u$ has not been considered in Theorem 4.1, since a MAP 2 defined by $\{x, y, z, u, w, v\}$ with $x>u$ is equivalent to the $\operatorname{MAP}_{2}\left\{x^{\prime}, y^{\prime}, z^{\prime}, u^{\prime}, w^{\prime}, v^{\prime}\right\}=\{u, z, y, x,-z-$ $u-v,-x-y-w\}$ with $x^{\prime}<u^{\prime}$, or, equivalently, the $\mathrm{MAP}_{2}$ can be parametrized by replacing state 1 by state 2 . 
Proof of Theorem 4.1. See Appendix D.

As a consequence of Theorem 4.1, the following two corollaries can be derived.

Corollary 4.1. Consider a $M A P_{2}$, $\mathcal{M}$, as in (3.1), and values $\tilde{u}, \tilde{z}, \tilde{x}(\tilde{u}, \tilde{z}), \tilde{y}(\tilde{u}, \tilde{z}), \tilde{v}(\tilde{u}, \tilde{z})$, and $\tilde{w}(\tilde{u}, \tilde{z})$ as in Proposition 4.2 characterizing another $M A P_{2}$, $\tilde{\mathcal{M}}$. Let $\boldsymbol{\Delta}(s)$ be defined as in (2.5) (define $\tilde{\boldsymbol{\Delta}}(s)$ similarly). Then,

$$
\begin{aligned}
\boldsymbol{\phi} \boldsymbol{\Delta}\left(s_{1}\right) \boldsymbol{e} & =\tilde{\boldsymbol{\phi}} \tilde{\boldsymbol{\Delta}}\left(s_{1}\right) \boldsymbol{e} \\
\boldsymbol{\phi} \boldsymbol{\Delta}\left(s_{1}\right) \boldsymbol{\Delta}\left(s_{2}\right) \boldsymbol{e} & =\tilde{\boldsymbol{\phi}} \tilde{\boldsymbol{\Delta}}\left(s_{1}\right) \tilde{\boldsymbol{\Delta}}\left(s_{2}\right) \boldsymbol{e} \\
(0,1) \boldsymbol{\Delta}\left(s_{1}\right) \boldsymbol{e} & =(0,1) \tilde{\boldsymbol{\Delta}}\left(s_{1}\right) \boldsymbol{e} \\
(0,1) \boldsymbol{\Delta}\left(s_{1}\right) \boldsymbol{\Delta}\left(s_{2}\right) \boldsymbol{e} & =(0,1) \tilde{\boldsymbol{\Delta}}\left(s_{1}\right) \tilde{\Delta}\left(s_{2}\right) \boldsymbol{e}
\end{aligned}
$$

for all $s_{1}, s_{2}$.

From (2.4), expressions (4.10)-(4.11) are an alternative way to state that (4.2) holds for $n=1$ and $n=2$. By substituting the values of $\mathcal{F}$ found in Theorem 4.1 in the expression for $\tilde{\boldsymbol{\Delta}}(s)($ see $(2.5))$, routine but tedious calculations yield (4.12)-(4.13).

Corollary 4.2. Consider a $M A P_{2}, \mathcal{M}$, as in (3.1), and values $\tilde{u}, \tilde{z}, \tilde{x}(\tilde{u}, \tilde{z}), \tilde{y}(\tilde{u}, \tilde{z}), \tilde{v}(\tilde{u}, \tilde{z})$, and $\tilde{w}(\tilde{u}, \tilde{z})$ as in Proposition 4.2 characterizing another $M A P_{2}, \tilde{\mathcal{M}}$. Let $\boldsymbol{\Delta}(s)$ be defined as in (2.5) (define $\tilde{\boldsymbol{\Delta}}(\mathrm{s})$ similarly). If $\boldsymbol{\Delta}(\mathrm{s})$ is given by

$$
\boldsymbol{\Delta}(s)=\left(\begin{array}{ll}
a(s) & b(s) \\
c(s) & d(s)
\end{array}\right)
$$

then the solution to (4.10)-(4.13) is

$$
\tilde{\Delta}(s)=\left(\begin{array}{ll}
\tilde{a}(s) & \tilde{b}(s) \\
\tilde{c}(s) & \tilde{d}(s)
\end{array}\right),
$$

where

$$
\begin{aligned}
& \tilde{a}(s)=\frac{\phi(a(s)-c(s))+\tilde{\phi} c(s)}{\phi} \\
& \tilde{b}(s)=\frac{\phi \tilde{\phi}(d(s)+2 c(s)-a(s))+\phi^{2}(a(s)-d(s)+b(s)-c(s))-\tilde{\phi}^{2} c(s)}{\phi \tilde{\phi}} \\
& \tilde{c}(s)=\frac{\tilde{\phi} c(s)}{\phi} \\
& \tilde{d}(s)=\frac{\phi(c(s)+d(s))-\tilde{\phi} c(s)}{\phi} .
\end{aligned}
$$

Equations (4.10)-(4.13) form a system with four equations, where the unknowns are the elements of (4.14). A trivial verification shows that (4.15)-(4.18) solves the system. The previous corollary motivates the following definition.

Definition 4.2. Let $\boldsymbol{G}$ and $\tilde{\boldsymbol{G}}$ be $2 \times 2$ matrices, where

$$
\boldsymbol{G}=\left(\begin{array}{ll}
a & b \\
c & d
\end{array}\right) \quad \text { and } \quad \tilde{\boldsymbol{G}}=\left(\begin{array}{cc}
\tilde{a} & \tilde{b} \\
\tilde{c} & \tilde{d}
\end{array}\right)
$$


It will be said that $\tilde{\boldsymbol{G}}$ is related to $\boldsymbol{G}$, given the values $\phi$ and $\tilde{\boldsymbol{\phi}}$, if and only if

$$
\begin{aligned}
& \tilde{a}=\frac{\phi(a-c)+\tilde{\phi} c}{\phi}, \\
& \tilde{b}=\frac{\phi \tilde{\phi}(d+2 c-a)+\phi^{2}(a-d+b-c)-\tilde{\phi}^{2} c}{\phi \tilde{\phi}}, \\
& \tilde{c}=\frac{\tilde{\phi} c}{\phi}, \\
& \tilde{d}=\frac{\phi(c+d)-\tilde{\phi} c}{\phi} .
\end{aligned}
$$

This relation will be denoted by $\boldsymbol{G} \stackrel{\phi, \tilde{\phi}}{\sim} \tilde{\boldsymbol{G}}$.

The following result is a direct consequence of the definition of $\tilde{\boldsymbol{G}},(4.19)-(4.22)$.

Proposition 4.3. If $\boldsymbol{G} \stackrel{\phi, \tilde{\phi}}{\sim} \tilde{\boldsymbol{G}}$ then

$$
\phi \boldsymbol{G} \boldsymbol{e}=\tilde{\phi} \tilde{\boldsymbol{G}} \boldsymbol{e},
$$

where $\phi=(\phi, 1-\phi)$ and $\tilde{\phi}=(\tilde{\phi}, 1-\tilde{\phi})$.

The proof follows straightforwardly from the definition of $\tilde{\boldsymbol{G}},(4.19)-(4.22)$.

The next result, whose proof can be found in Appendix E, is crucial for proving the nonidentifiability of the $\mathrm{MAP}_{2}$.

Proposition 4.4. If $\boldsymbol{G} \stackrel{\phi, \tilde{\phi}}{\sim} \tilde{\boldsymbol{G}}$ and $\boldsymbol{H} \stackrel{\phi, \tilde{\phi}}{\sim} \tilde{\boldsymbol{H}}$, then

$$
\boldsymbol{G H} \stackrel{\phi, \tilde{\phi}}{\sim} \tilde{\boldsymbol{G}} \tilde{\boldsymbol{H}} .
$$

Finally, we can prove the general theorem.

Theorem 4.2. The $M A P_{2}$ is not an identifiable process.

Proof. The proof is based on the fact that, given a $\mathrm{MAP}_{2}, \mathcal{M}$, as in (3.1), any other $\mathrm{MAP}_{2}$, $\tilde{\mathcal{M}}$, chosen from the set $\mathcal{F}$ (see Theorem 4.1) satisfies equality (4.2) for all $n$.

If $\tilde{\mathcal{M}}$ is selected from $\mathcal{F}$ then, from Corollary 4.1 ,

$$
\boldsymbol{\Delta}\left(s_{1}\right) \stackrel{\phi, \tilde{\phi}}{\sim} \tilde{\boldsymbol{\Delta}}\left(s_{1}\right) \quad \text { and } \quad \boldsymbol{\Delta}\left(s_{1}\right) \boldsymbol{\Delta}\left(s_{2}\right) \stackrel{\phi, \tilde{\phi}}{\sim} \tilde{\boldsymbol{\Delta}}\left(s_{1}\right) \tilde{\boldsymbol{\Delta}}\left(s_{2}\right) \text { for all } s_{1}, s_{2} .
$$

We conclude from Proposition 4.4 that

$$
\boldsymbol{\Delta}\left(s_{1}\right) \boldsymbol{\Delta}\left(s_{2}\right) \boldsymbol{\Delta}\left(s_{3}\right) \stackrel{\phi, \tilde{\phi}}{\sim} \tilde{\boldsymbol{\Delta}}\left(s_{1}\right) \tilde{\boldsymbol{\Delta}}\left(s_{2}\right) \tilde{\Delta}\left(s_{3}\right) \text { for all } s_{1}, s_{2}, s_{3},
$$

and, finally,

$$
\prod_{i=1}^{n} \boldsymbol{\Delta}\left(s_{i}\right) \stackrel{\phi, \tilde{\phi}}{\sim} \prod_{i=1}^{n} \tilde{\boldsymbol{\Delta}}\left(s_{i}\right),
$$

which, by Proposition 4.3, is equivalent to (4.2) for all $n \geq 1$.

To illustrate Theorem 4.2, we provide an example showing two $\mathrm{MAP}_{2} \mathrm{~s}$ that verify (4.2) for all $n \geq 1$. 
Example 4.1. Consider the $\mathrm{MAP}_{2}$ defined by

$$
\boldsymbol{D}_{0}=\left(\begin{array}{cc}
-10 & 2.5 \\
0.6 & -3
\end{array}\right), \quad \boldsymbol{D}_{1}=\left(\begin{array}{cc}
4 & 3.5 \\
1.35 & 1.05
\end{array}\right)
$$

or, alternatively,

$$
\boldsymbol{P}_{0}=\left(\begin{array}{cc}
0 & 0.25 \\
0.2 & 0
\end{array}\right), \quad \boldsymbol{P}_{1}=\left(\begin{array}{cc}
0.4 & 0.35 \\
0.45 & 0.35
\end{array}\right)
$$

with exponential rates $\left(\lambda_{1}, \lambda_{2}\right)=(10,3)$ and stationary probability distribution

$$
\phi=(0.5474,0.4526) \text {. }
$$

It can be seen that $\varepsilon$, defined in (4.8), has to be chosen from

$$
0<\varepsilon<\min \{10,3.5,0.4961,7.2081,0.4667,0.5120\} .
$$

Let $\varepsilon=0.3$. Then, according to the equations given in Theorem 4.1,

$$
\tilde{\boldsymbol{D}}_{0}=\left(\begin{array}{cc}
-9.7 & 3.9 \\
0.9 & -3.3
\end{array}\right), \quad \tilde{\boldsymbol{D}}_{1}=\left(\begin{array}{cc}
4.675 & 1.1250 \\
2.025 & 0.375
\end{array}\right),
$$

or

$$
\tilde{\boldsymbol{P}}_{0}=\left(\begin{array}{cc}
0 & 0.4020 \\
0.2727 & 0
\end{array}\right), \quad \tilde{\boldsymbol{P}}_{1}=\left(\begin{array}{ll}
0.4820 & 0.1160 \\
0.6137 & 0.1136
\end{array}\right),
$$

with exponential rates $\left(\tilde{\lambda}_{1}, \tilde{\lambda}_{2}\right)=(9.7,3.3)$ and stationary distribution $\tilde{\boldsymbol{\phi}}=(0.8217,0.1783)$. Because of the proof of Theorem 4.2, (4.2) holds for all $s$ and $n$, that is, both $\mathrm{MAP}_{2} \mathrm{~s}$ will possess the same joint interarrival time distribution.

Example 4.2. We consider in this example a $\mathrm{MAP}_{2}$ such that $x=u$, defined by

$$
\boldsymbol{D}_{0}=\left(\begin{array}{cc}
-3 & 0.9 \\
0.6 & -3
\end{array}\right), \quad \boldsymbol{D}_{1}=\left(\begin{array}{cc}
0.9 & 1.2 \\
1.5 & 0.9
\end{array}\right)
$$

or, alternatively,

$$
\boldsymbol{P}_{0}=\left(\begin{array}{cc}
0 & 0.3 \\
0.2 & 0
\end{array}\right), \quad \boldsymbol{P}_{1}=\left(\begin{array}{cc}
0.3 & 0.4 \\
0.5 & 0.3
\end{array}\right),
$$

with exponential rates $\left(\lambda_{1}, \lambda_{2}\right)=(3,3)$ and stationary probability distribution

$$
\boldsymbol{\phi}=(0.5333,0.4667) \text {. }
$$

In this case $\varepsilon$, defined in (4.9), has to be chosen from

$$
0<\varepsilon<\min \{3,0.5250,0.7348,0.36,0.5367\} \text {. }
$$

Set, for example, $\varepsilon=0.15$. Then, according to the equations given in Theorem 4.1,

$$
\tilde{\boldsymbol{D}}_{0}=\left(\begin{array}{cc}
-2.85 & 0.69 \\
0.75 & -3.15
\end{array}\right), \quad \tilde{\boldsymbol{D}}_{1}=\left(\begin{array}{cc}
1.275 & 0.885 \\
1.875 & 0.5250
\end{array}\right),
$$

or

$$
\tilde{\boldsymbol{P}}_{0}=\left(\begin{array}{cc}
0 & 0.2421 \\
0.2381 & 0
\end{array}\right), \quad \tilde{\boldsymbol{P}}_{1}=\left(\begin{array}{ll}
0.4474 & 0.3105 \\
0.5952 & 0.1667
\end{array}\right),
$$

with exponential rates $\left(\tilde{\lambda}_{1}, \tilde{\lambda}_{2}\right)=(3.15,2.85)$ and stationary distribution

$$
\tilde{\boldsymbol{\phi}}=(0.6667,0.3333) \text {, }
$$

is equivalent to $\left\{\boldsymbol{D}_{0}, \boldsymbol{D}_{1}\right\}$. 
Some remarks need to be made here. First, let us point out that, as has been described in the proof of Theorem 4.2, given a fixed $\mathrm{MAP}_{2}, \mathcal{M}$, any other $\mathrm{MAP}_{2}, \tilde{\mathcal{M}}$, chosen from the set $\mathcal{F}$ will verify (4.2) for all $s$ and $n$, and, thus, both $\mathrm{MAP}_{2} \mathrm{~s}$ will have the same joint interarrival time distribution.

Our second remark is connected with the MMPP. Its definition implies that $w \equiv-x-y$ and $v \equiv 0$. As Rydén [23] showed, the MMPP is an identifiable process up to permutations of the states, or, equivalently, for the two-states case, if the set $\{x, y, z, u, w=-x-y, v=0\}$ defines an $\mathrm{MMPP}_{2}$ then the only $\mathrm{MMPP}_{2}$ with the same likelihood will be given by

$$
\{\tilde{x}=u, \tilde{y}=z, \tilde{z}=y, \tilde{u}=x, \tilde{w}=-z-u, \tilde{v}=0\} .
$$

It can be verified that, when $\tilde{v}=v=0, \tilde{w}=-\tilde{x}-\tilde{y}$, and $w=-x-y$, Proposition 4.2 provides just two solutions, the original $\mathrm{MMPP}_{2}$ and its permuted version, given by (4.23). Thus, our results are equivalent to Rydén's.

From the nonidentifiability of the $\mathrm{MAP}_{2}$ and the identifiability of the $\mathrm{MMPP}_{2}$, one could wonder if, given a MAP 2 , there exists an equivalent $\mathrm{MMPP}_{2}$. This has been tested from the equations given in Proposition 4.2, and the answer is that this is not true, in general. The following example illustrates this fact.

Example 4.3. Let us consider the same $\mathrm{MAP}_{2}$ defined in Example 1:

$$
\boldsymbol{D}_{0}=\left(\begin{array}{cc}
-10 & 2.5 \\
0.6 & -3
\end{array}\right), \quad \boldsymbol{D}_{1}=\left(\begin{array}{cc}
4 & 3.5 \\
1.35 & 1.05
\end{array}\right) \text {. }
$$

Then, according to the solutions given in Proposition 4.2 (having previously fixed $w=-x-y$ and $v=0$ ), the only equivalent $\mathrm{MMPP}_{2}$ is given by

$$
\tilde{D}_{0}=\left(\begin{array}{cc}
-7.4231 & 2.2712 \\
5.6788 & -5.5769
\end{array}\right), \quad \tilde{D}_{1}=\left(\begin{array}{cc}
5.1519 & 0 \\
0 & -0.1019
\end{array}\right),
$$

which, since $d_{221}<0$, does not define a real MMPP 2 . Thus, in this case, there does not exist an $\mathrm{MMPP}_{2}$ equivalent to the given $\mathrm{MAP}_{2}$. However, there do exist $\mathrm{MAP}_{2}$ s that can be reduced to $\mathrm{MMPP}_{2}$ s. For example,

$$
\boldsymbol{D}_{0}=\left(\begin{array}{cc}
-20 & 8 \\
3.5 & -5
\end{array}\right), \quad \boldsymbol{D}_{1}=\left(\begin{array}{cc}
11.5 & 0.5 \\
0.5 & 1
\end{array}\right)
$$

define a $\mathrm{MAP}_{2}$ that is equivalent to the $\mathrm{MMPP}_{2}$ defined by

$$
\tilde{\boldsymbol{D}}_{0}=\left(\begin{array}{cc}
-19.4211 & 7.8973 \\
4.6027 & -5.5789
\end{array}\right), \quad \tilde{\boldsymbol{D}}_{1}=\left(\begin{array}{cc}
11.5238 & 0 \\
0 & 0.9762
\end{array}\right) .
$$

Finally, we discuss the case of phase-type $(\mathrm{PH})$ renewal processes, a special type of MAP. It is known (see, for example, [15]) that a PH renewal process with representation $(\boldsymbol{\eta}, \boldsymbol{T})$ is a MAP, where $\boldsymbol{D}_{0}=\boldsymbol{T}$ and $\boldsymbol{D}_{1}=-\boldsymbol{T} \boldsymbol{e} \boldsymbol{\eta}$. If we focus on two-state MAPs, defined by (3.1), this implies that

$$
\eta=-\frac{w}{x+y}=-\frac{v}{u+z}
$$

where $\boldsymbol{\eta}=(\eta, 1-\eta)$, and, thus, for a PH renewal process,

$$
v=\frac{w(z+u)}{x+y}
$$


Example 4.4. As an example, consider the $\mathrm{PH}$ process given by

$$
\boldsymbol{T}=\left(\begin{array}{cc}
-10 & 2.5 \\
0.6 & -3
\end{array}\right), \quad \boldsymbol{\eta}=(0.4,0.6)
$$

Then, the process defined by (4.26) can be also expressed as a $\mathrm{MAP}_{2}$ as

$$
\boldsymbol{D}_{0}=\left(\begin{array}{cc}
-10 & 2.5 \\
0.6 & -3
\end{array}\right), \quad \boldsymbol{D}_{1}=\left(\begin{array}{cc}
3 & 4.5 \\
0.96 & 1.44
\end{array}\right)
$$

where it can be easily seen that (4.25) holds. Conversely, given the $\mathrm{MAP}_{2}$

$$
\boldsymbol{D}_{0}=\left(\begin{array}{cc}
-5 & 1.4 \\
0.25 & -0.5
\end{array}\right), \quad \boldsymbol{D}_{1}=\left(\begin{array}{cc}
2.52 & 1.08 \\
0.175 & 0.075
\end{array}\right)
$$

it can be checked that (4.25) holds, and, therefore, (4.27) admits a PH representation $(\boldsymbol{\eta}, \boldsymbol{T})$, where $\boldsymbol{T}=\boldsymbol{D}_{0}$ and $\boldsymbol{\eta}$ is computed from (4.24) as $\boldsymbol{\eta}=(0.7,0.3)$.

In this setting, given a fixed two-state PH renewal process, it is of interest to know the set of equivalent $\mathrm{MAP}_{2} \mathrm{~s}$, which implies deriving expressions (4.4)-(4.7), assuming that $v=$ $w(z+u) /(x+y)$. It follows immediately that if $v=w(z+u) /(x+y)$ then

$$
\{\tilde{u}, \tilde{z}, \tilde{x}(\tilde{u}, \tilde{z}), \tilde{y}(\tilde{u}, \tilde{z}), \tilde{v}(\tilde{u}, \tilde{z}), \tilde{w}(\tilde{u}, \tilde{z})\}
$$

computed according to Proposition 4.2, also verifies that $\tilde{v}=\tilde{w}(\tilde{z}+\tilde{u}) /(\tilde{x}+\tilde{y})$. This implies that the $\mathrm{MAP}_{2}$ s equivalent to a given two-state $\mathrm{PH}$ renewal process are also $\mathrm{PH}$ renewal processes. Another interesting fact with regards to two-state $\mathrm{PH}$ renewal processes is that, since $v=$ $w(z+u) /(x+y)$, it can be verified that $\varepsilon_{3}=\varepsilon_{5}=\varepsilon_{6}$ (see Theorem 4.1). In the following example, some $\mathrm{MAP}_{2} \mathrm{~s}$, equivalent to a given two-state $\mathrm{PH}$ distribution, are computed.

Example 4.5. Consider the two-state PH renewal process defined by (4.27). Then, in order to find the equivalent $\mathrm{MAP}_{2} \mathrm{~s}$, the value of $\varepsilon$ in Theorem 4.1 has to be chosen from

$$
0<\varepsilon<\{5,2.25,0.1071,4.5765,0.1071,0.1071\}
$$

Note that, indeed, $\varepsilon_{3}=\varepsilon_{5}=\varepsilon_{6}$. Let $\varepsilon=0.05$. Then, an equivalent $\mathrm{MAP}_{2}$ is obtained as

$$
\tilde{\boldsymbol{D}}_{0}=\left(\begin{array}{cc}
-4.95 & 1.9083 \\
0.3 & -0.55
\end{array}\right), \quad \tilde{\boldsymbol{D}}_{1}=\left(\begin{array}{cc}
2.555 & 0.4867 \\
0.21 & 0.04
\end{array}\right)
$$

where it can be easily seen that (4.25) holds, and, thus, the generated equivalent $\mathrm{MAP}_{2}$ is a $\mathrm{PH}$ renewal process given by $(\tilde{\boldsymbol{\eta}}, \tilde{\boldsymbol{T}})$, where $\tilde{\boldsymbol{T}}=\tilde{\boldsymbol{D}}_{0}$ and $\tilde{\boldsymbol{\eta}}=(0.84,0.16)$. If instead $\varepsilon=0.09$ is chosen, the obtained equivalent $\mathrm{MAP}_{2}$ is

$$
\tilde{\boldsymbol{D}}_{0}=\left(\begin{array}{cc}
-4.9 & 2.2571 \\
0.35 & -0.6
\end{array}\right), \quad \tilde{\boldsymbol{D}}_{1}=\left(\begin{array}{cc}
2.59 & 0.0529 \\
0.245 & 0.05
\end{array}\right),
$$

with PH representation $(\tilde{\boldsymbol{\eta}}, \tilde{\boldsymbol{T}})$, where $\tilde{\boldsymbol{T}}=\tilde{\boldsymbol{D}}_{0}$ and $\tilde{\boldsymbol{\eta}}=(0.98,0.02)$. 


\section{Conclusions and extensions}

In spite of the good properties that the MAPs present, which make them very suitable processes for modeling nonexponential events, there exist few works dealing with the identifiability of the MAP, which is of crucial importance when inference for the process is considered.

In this work we have provided a procedure that shows, for any fixed $\mathrm{MAP}_{2}$, how to build another, equivalent $\mathrm{MAP}_{2}$. We have also shown that if two $\mathrm{MAP}_{2} \mathrm{~s}$ have equal LaplaceStieltjes transform (LST) for one and two data, then their LST will be equal for any set of points $\left(s_{1}, \ldots, s_{n}\right)$. Calculations have been carried out using MATLAB ${ }^{\circledR}$, and all code utilized in the examples is available from the authors on request.

A number of extensions are possible. Firstly, we could consider the batch $\mathrm{MAP}_{2}$, where batch arrivals are permitted. Furthermore, we could extend this analysis to MAPs or BMAPs with more than two states. Finally, it is of practical interest to consider what happens when there is missing data, that is, when a full sequence of interarrival times is not considered. Work on these problems is underway.

\section{Appendix A. Proof of (2.3)}

As $\phi$ is the unique solution to $\boldsymbol{\phi} \boldsymbol{P}^{\star}=\boldsymbol{\phi}$, we need to show that

$$
\left(\pi D_{1} e\right)^{-1} \pi D_{1} P^{\star}=\left(\pi D_{1} e\right)^{-1} \pi D_{1} .
$$

Define $\boldsymbol{\Lambda}=\operatorname{diag}\left\{\lambda_{1}, \ldots, \lambda_{m}\right\}$, that is, a diagonal $m \times m$ matrix with diagonal equal to $\left(\lambda_{1}, \ldots, \lambda_{m}\right)$. Then $\boldsymbol{D}_{1}=\boldsymbol{\Lambda} \boldsymbol{P}_{1}$ and $\boldsymbol{D}_{0}=\boldsymbol{\Lambda}\left(\boldsymbol{P}_{0}-\boldsymbol{I}\right)$. From $(2.2), \boldsymbol{P}^{\star}=\left(-\boldsymbol{\Lambda}^{-1} \boldsymbol{D}_{0}\right)^{-1} \boldsymbol{\Lambda}^{-1} \boldsymbol{D}_{1}$, and, thus,

$$
\begin{aligned}
\left(\pi D_{1} e\right)^{-1} \pi D_{1} P^{\star} & =\left(\pi D_{1} e\right)^{-1} \pi D_{1}\left(-\Lambda^{-1} D_{0}\right)^{-1} \Lambda^{-1} D_{1} \\
& =-\left(\pi D_{1} e\right)^{-1} \pi D_{1} D_{0}^{-1} D_{1} \\
& =-\left(\pi D_{1} e\right)^{-1} \pi\left(D-D_{0}\right) D_{0}^{-1} D_{1} \\
& =-\left(\pi D_{1} e\right)^{-1}\left(\pi D-\pi D_{0}\right) D_{0}^{-1} D_{1} \\
& =\left(\pi D_{1} e\right)^{-1} \pi D_{1}
\end{aligned}
$$

\section{Appendix B. Proof of Theorem 3.1}

Firstly, we provide some lemmas that will be necessary for the proof.

Lemma B.1. Let $T_{n}$ and $\tilde{T}_{n}$ denote the times between the $(n-1)$ th and $n$th arrival in two $M A P_{2} s, \mathcal{M}$ and $\tilde{\mathcal{M}}$. Then,

$$
T_{n} \stackrel{\mathrm{D}}{=} \tilde{T}_{n} \text { for all } n \geq 1
$$

if and only if

$$
\boldsymbol{\theta}\left(\boldsymbol{P}^{\star}\right)^{(n-1)}\left(s \boldsymbol{I}-\boldsymbol{D}_{0}\right)^{-1} \boldsymbol{D}_{1} \boldsymbol{e}=\tilde{\boldsymbol{\theta}}\left(\tilde{\boldsymbol{P}}^{\star}\right)^{(n-1)}\left(s \boldsymbol{I}-\tilde{\boldsymbol{D}}_{0}\right)^{-1} \tilde{\boldsymbol{D}}_{1} \boldsymbol{e}
$$

for all $n \geq 1$ and all $s$.

Proof. The variables $T_{n}$ and $\tilde{T}_{n}$ are equally distributed if and only if their Laplace transforms are the same. These are given by (B.1), where $\boldsymbol{\theta}\left(\boldsymbol{P}^{\star}\right)^{(n-1)}$ and $\tilde{\boldsymbol{\theta}}\left(\tilde{\boldsymbol{P}}^{\star}\right)^{(n-1)}$ represent the 'initial' probabilities after $n-1$ arrivals.

A similar result to Lemma B.1, which provides a different characterization of condition (C1) in Theorem 3.1, is shown next. 
Lemma B.2. Let $T$ and $\tilde{T}$ denote the interarrival times of two stationary $M A P_{2} s, \mathcal{M}$ and $\tilde{\mathcal{M}}$, with stationary probabilities $\boldsymbol{\phi}$ and $\tilde{\boldsymbol{\phi}}$. Then,

$$
T \stackrel{\mathrm{D}}{=} \tilde{T}
$$

if and only if

$$
\boldsymbol{\phi}\left(s \boldsymbol{I}-\boldsymbol{D}_{0}\right)^{-1} \boldsymbol{D}_{1} \boldsymbol{e}=\tilde{\boldsymbol{\phi}}\left(s \boldsymbol{I}-\tilde{\boldsymbol{D}}_{0}\right)^{-1} \tilde{\boldsymbol{D}}_{1} \boldsymbol{e} .
$$

Proof. We proceed as in the proof of Lemma B.1, but taking into account the fact that

$$
\lim _{n \rightarrow \infty}\left(\boldsymbol{P}^{\star}\right)^{n}=\boldsymbol{\Phi}, \quad \lim _{n \rightarrow \infty}\left(\tilde{\boldsymbol{P}}^{\star}\right)^{n}=\tilde{\boldsymbol{\Phi}},
$$

or, equivalently, $\lim _{n \rightarrow \infty} \boldsymbol{\theta}\left(\boldsymbol{P}^{\star}\right)^{n}=\boldsymbol{\phi}$ and $\lim _{n \rightarrow \infty} \tilde{\boldsymbol{\theta}}\left(\tilde{\boldsymbol{P}}^{\star}\right)^{n}=\tilde{\boldsymbol{\phi}}$.

Lemma B.3. Let $\mathcal{M}$ and $\tilde{\mathcal{M}}$ denote two $M A P_{2}$ s, and let $\rho=(\rho, 1-\rho), \tilde{\rho}=(\tilde{\rho}, 1-\tilde{\rho})$ be any probability vectors. If

$$
\boldsymbol{\rho}\left(s \boldsymbol{I}-\boldsymbol{D}_{0}\right)^{-1} \boldsymbol{D}_{1} \boldsymbol{e}=\tilde{\boldsymbol{\rho}}\left(s \boldsymbol{I}-\tilde{\boldsymbol{D}}_{0}\right)^{-1} \tilde{\boldsymbol{D}}_{1} \boldsymbol{e}
$$

then

$$
c \rho+\tilde{c} \tilde{\rho}+d=0
$$

where $c$ and $\tilde{c}$ are defined as in (3.4), and $d=\tilde{z}+\tilde{u}-z-u$.

Proof. Substituting $\boldsymbol{D}_{0}, \boldsymbol{D}_{1}, \tilde{\boldsymbol{D}}_{0}$, and $\tilde{\boldsymbol{D}}_{1}$ expressed as in (3.1), it can be seen that

$$
\boldsymbol{\rho}\left(s \boldsymbol{I}-\boldsymbol{D}_{0}\right)^{-1} \boldsymbol{D}_{1} \boldsymbol{e}=\frac{\alpha s+\gamma}{s^{2}+\beta s+\gamma},
$$

where the coefficients $\alpha, \beta$, and $\gamma$ are

$$
\alpha=\rho(z+u-x-y)-(z+u), \quad \beta=-x-u, \quad \gamma=x u-y z .
$$

Similarly,

$$
\tilde{\boldsymbol{\rho}}\left(s \boldsymbol{I}-\boldsymbol{D}_{0}\right)^{-1} \boldsymbol{D}_{1} \boldsymbol{e}=\frac{\tilde{\alpha} s+\tilde{\gamma}}{s^{2}+\tilde{\beta} s+\tilde{\gamma}},
$$

where

$$
\tilde{\alpha}=\tilde{\rho}(\tilde{z}+\tilde{u}-\tilde{x}-\tilde{y})-(\tilde{z}+\tilde{u}), \quad \tilde{\beta}=-\tilde{x}-\tilde{u}, \quad \tilde{\gamma}=\tilde{x} \tilde{u}-\tilde{y} \tilde{z} .
$$

Next, if

$$
\frac{\alpha s+\gamma}{s^{2}+\beta s+\gamma}=\frac{\tilde{\alpha} s+\tilde{\gamma}}{s^{2}+\tilde{\beta} s+\tilde{\gamma}}
$$

then it can easily be seen that

$$
\alpha=\tilde{\alpha},
$$

which is equivalent, given the definitions of $\alpha$ and $\tilde{\alpha}$, to

$$
c \rho+\tilde{c} \tilde{\rho}+d=0
$$

with $c$ and $\tilde{c}$ as in (3.4), and $d=\tilde{z}+\tilde{u}-z-u$. 
Lemma B.4. Let $\boldsymbol{P}^{\star}$ be the transition probability matrix in a $M A P_{2}$ with vector of stationary probabilities $\boldsymbol{\phi}$. If all the rows of $\boldsymbol{P}^{\star}$ are equal then $\boldsymbol{P}^{\star}=\Phi$, the matrix with all rows equal to $\phi$.

Proof. The proof is straightforward once the equation $\boldsymbol{\phi} \boldsymbol{P}^{\star}=\boldsymbol{\phi}$ is solved, where it is assumed that all the rows of $\boldsymbol{P}^{\star}$ are equal.

Finally, if $\boldsymbol{P}^{\star}$ is a transition matrix with vector of stationary probabilities $\boldsymbol{\phi}$, where

$$
\boldsymbol{P}^{\star}=\left(\begin{array}{ll}
p_{11}^{\star} & p_{12}^{\star} \\
p_{21}^{\star} & p_{22}^{\star}
\end{array}\right),
$$

and $\phi=(\phi, 1-\phi)$, then it is straightforward to check that

$$
\phi=\frac{p_{21}^{\star}}{1-p_{11}^{\star}+p_{21}^{\star}} .
$$

Proof of Theorem 3.1. Conditions $(\mathrm{Cl})$ and $(\mathrm{C} 2)$ imply weak convergence. Let us first assume that both $(\mathrm{C} 1)$ and $(\mathrm{C} 2)$ hold. We want to prove equivalence given by (B.1) for all $s$ and $n \geq 1$. By Lemma B.2, condition (C1) is equivalent to (B.2). But, since $(\boldsymbol{\theta}, \tilde{\boldsymbol{\theta}})=(\boldsymbol{\phi}, \tilde{\boldsymbol{\phi}})$, $\boldsymbol{\theta} \boldsymbol{P}^{\star}=\boldsymbol{\phi} \boldsymbol{P}^{\star}=\boldsymbol{\phi}$, and $\tilde{\boldsymbol{\theta}} \tilde{\boldsymbol{P}}^{\star}=\tilde{\boldsymbol{\phi}} \tilde{\boldsymbol{P}}^{\star}=\tilde{\boldsymbol{\phi}}$, then (B.2) becomes (B.1) and, thus, weak equivalence is obtained.

Weak convergence implies conditions $(C 1)$ and (C2). If two given $\mathrm{MAP}_{2} \mathrm{~s}$ are weakly equivalent, (B.1) holds for all $s$ and $n \geq 1$. If $n \rightarrow \infty$ then, from Lemma B.2, (B.2) holds, and, thus, (C1) holds too. Let us deduce (C2) from weak equivalence; since (B.2) holds, then the pair $(\boldsymbol{\phi}, \tilde{\boldsymbol{\phi}})$ verifies (B.3) (where $\rho=\phi$ and $\tilde{\rho}=\tilde{\phi}$ ), that is,

$$
c \phi+\tilde{c} \tilde{\phi}+d=0 .
$$

Because of weak equivalence, (B.1) holds for $n=1$, and, thus, the pair $(\boldsymbol{\theta}, \tilde{\boldsymbol{\theta}})$ also satisfies (B.3), i.e.

$$
c \theta+\tilde{c} \tilde{\theta}+d=0
$$

Both equations imply that

$$
c \phi+\tilde{c} \tilde{\phi}=c \theta+\tilde{c} \tilde{\theta}
$$

or, equivalently, using (B.5),

$$
c \frac{p_{21}^{\star}}{1-p_{11}^{\star}+p_{21}^{\star}}+\tilde{c} \frac{\tilde{p}_{21}^{\star}}{1-\tilde{p}_{11}^{\star}+\tilde{p}_{21}^{\star}}=c \theta+\tilde{c} \tilde{\theta} .
$$

Again, because of weak equivalence, and taking $n=2$ in condition (B.1), then

$$
c \frac{p_{21}^{\star}}{1-p_{11}^{\star}+p_{21}^{\star}}+\tilde{c} \frac{\tilde{p}_{21}^{\star}}{1-\tilde{p}_{11}^{\star}+\tilde{p}_{21}^{\star}}=c \theta^{(1)}+\tilde{c} \tilde{\theta}^{(1)},
$$

where

$$
\boldsymbol{\theta}^{(1)}=\boldsymbol{\theta} \boldsymbol{P}^{\star}=\left(\theta^{(1)}, 1-\theta^{(1)}\right), \quad \tilde{\boldsymbol{\theta}}^{(1)}=\tilde{\boldsymbol{\theta}} \tilde{\boldsymbol{P}}^{\star}=\left(\tilde{\theta}^{(1)}, 1-\tilde{\theta}^{(1)}\right) .
$$

It can be checked that

$$
\theta^{(1)}=\theta\left(p_{11}^{\star}-p_{21}^{\star}\right)+p_{21}^{\star}, \quad \tilde{\theta}^{(1)}=\tilde{\theta}\left(\tilde{p}_{11}^{\star}-\tilde{p}_{21}^{\star}\right)+\tilde{p}_{21}^{\star},
$$


and, thus, we need to solve for $(\theta, \tilde{\theta})$ in the following system of linear equations:

$$
\begin{gathered}
c \theta+\tilde{c} \tilde{\theta}=c \frac{p_{21}^{\star}}{1-p_{11}^{\star}+p_{21}^{\star}}+\tilde{c} \frac{\tilde{p}_{21}^{\star}}{1-\tilde{p}_{11}^{\star}+\tilde{p}_{21}^{\star},} \\
c\left(p_{11}^{\star}-p_{21}^{\star}\right) \theta+\tilde{c}\left(\tilde{p}_{11}^{\star}-\tilde{p}_{21}^{\star}\right) \tilde{\theta}=c\left(\frac{p_{21}^{\star}}{1-p_{11}^{\star}+p_{21}^{\star}}-p_{21}^{\star}\right)+\tilde{c}\left(\frac{\tilde{p}_{21}^{\star}}{1-\tilde{p}_{11}^{\star}+\tilde{p}_{21}^{\star}}-\tilde{p}_{21}^{\star}\right),
\end{gathered}
$$

whose coefficient matrix is

$$
\boldsymbol{C}=\left(\begin{array}{cc}
c & \tilde{c} \\
c\left(p_{11}^{\star}-p_{21}^{\star}\right) & \tilde{c}\left(\tilde{p}_{11}^{\star}-\tilde{p}_{21}^{\star}\right)
\end{array}\right) .
$$

It can be easily seen that $\boldsymbol{\theta}=\boldsymbol{\phi}$, and $\tilde{\boldsymbol{\theta}}=\tilde{\boldsymbol{\phi}}$ solves the system. We need to determine the uniqueness of this solution. This comes from (A1), (A2), and Lemma B.4; since $\boldsymbol{P}^{\star} \neq \boldsymbol{\Phi}$ or $\tilde{\boldsymbol{P}}^{\star} \neq \tilde{\boldsymbol{\Phi}}$, then, by Lemma B.4, either the rows of $\boldsymbol{P}^{\star}$ or that of $\tilde{\boldsymbol{P}}^{\star}$ are not equal. This implies that $p_{11}^{\star}-p_{21}^{\star} \neq 0$ or $\tilde{p}_{11}^{\star}-\tilde{p}_{21}^{\star} \neq 0$. In addition, since $c, \tilde{c} \neq 0$, the rank of $\boldsymbol{C}$ is 2 , and the solution is unique: $\boldsymbol{\theta}=\boldsymbol{\phi}$ and $\tilde{\boldsymbol{\theta}}=\tilde{\boldsymbol{\phi}}$.

\section{Appendix C. Proof of Proposition 4.1}

Let us first consider the case when $n=1$. It is known from the proof of Lemma B.2 that the equality of Laplace transforms $f_{T ; \boldsymbol{D}_{0}, \boldsymbol{D}_{1}}^{*}(s)=f_{\tilde{T} ; \tilde{\boldsymbol{D}}_{0}, \tilde{\boldsymbol{D}}_{1}}^{*}(s)$ in the stationary version is equivalent to (B.4), where $\boldsymbol{\rho}=\boldsymbol{\phi}$ and $\tilde{\boldsymbol{\rho}}=\tilde{\boldsymbol{\phi}}$. If $\tilde{\alpha}=\alpha, \tilde{\beta}=\beta$, and $\tilde{\gamma}=\gamma$, or, equivalently,

$$
\begin{aligned}
\tilde{\phi}(\tilde{z}+\tilde{u}-\tilde{x}-\tilde{y})-(\tilde{z}+\tilde{u}) & =\alpha, \\
-\tilde{x}-\tilde{u} & =\beta, \\
\tilde{x} \tilde{u}-\tilde{y} \tilde{z} & =\gamma,
\end{aligned}
$$

then (B.4) holds, and, thus, $\mathcal{M}$ and $\tilde{\mathcal{M}}$ are weakly equivalent.

In the two data case, it can be shown that

$$
f_{T ; \boldsymbol{D}_{0}, \boldsymbol{D}_{1}}^{*}\left(s_{1}, s_{2}\right)=\frac{\delta_{1} s_{1} s_{2}+\delta_{2} s_{2}+\alpha \gamma s_{1}+\gamma^{2}}{s_{1}^{2} s_{2}^{2}+\gamma s_{1}^{2}+\gamma s_{2}^{2}+\beta s_{1}^{2} s_{2}+\beta s_{1} s_{2}^{2}+\beta^{2} s_{1} s_{2}+\beta \gamma s_{2}+\gamma^{2}},
$$

where

$$
\begin{aligned}
\delta_{1}= & \phi\left((z+u-x-y)(w-v)+(x+y)(z+u)-(z+u)^{2}\right)+(z+u-x-y) v \\
& +(z+u)^{2}, \\
\delta_{2}= & \phi(x+y-z-u)(u w-y v-x v+z w)+(x+y-z-u)(x v-z w)-(u+z) \gamma .
\end{aligned}
$$

If (C.1)-(C.3) are satisfied, and, in addition,

$$
\begin{aligned}
& \tilde{\phi}\left((\tilde{z}+\tilde{u}-\tilde{x}-\tilde{y})(\tilde{w}-\tilde{v})+(\tilde{x}+\tilde{y})(\tilde{z}+\tilde{u})-(\tilde{z}+\tilde{u})^{2}\right)+(\tilde{z}+\tilde{u}-\tilde{x}-\tilde{y}) \tilde{v}+(\tilde{z}+\tilde{u})^{2} \\
& \quad=\delta_{1} \\
& \tilde{\phi}(\tilde{x}+\tilde{y}-\tilde{z}-\tilde{u})(\tilde{u} \tilde{w}-\tilde{y} \tilde{v}-\tilde{x} \tilde{v}+\tilde{z} \tilde{w})+(\tilde{x}+\tilde{y}-\tilde{z}-\tilde{u})(\tilde{x} \tilde{v}-\tilde{z} \tilde{w})-(\tilde{u}+\tilde{z}) \tilde{\gamma} \\
& \quad=\delta_{2}
\end{aligned}
$$

then (4.2) holds for $n=1$ and $n=2$. 


\section{Appendix D. Proof of Theorem 4.1}

The set $\mathcal{F}$ is contained in the set of solutions given by Proposition 4.2. To prove that the set $\mathcal{F}$ provides feasible solutions (real $\mathrm{MAP}_{2} \mathrm{~s}$ ), let us first assume that $x<u$. Let $\varepsilon$ be defined as in (4.8), that is,

$$
0<\varepsilon<\min \left\{\varepsilon_{1}, \varepsilon_{2}, \varepsilon_{3}, \varepsilon_{4}, \varepsilon_{5}, \varepsilon_{6}\right\} .
$$

We begin by proving that $\min \left\{\varepsilon_{1}, \varepsilon_{2}, \varepsilon_{3}, \varepsilon_{4}, \varepsilon_{5}, \varepsilon_{6}\right\}>0$. It is easily checked that

$$
\min \left\{\varepsilon_{1}, \varepsilon_{2}, \varepsilon_{3}, \varepsilon_{4}\right\}>0 .
$$

In addition, $\varepsilon_{5}=-(z / v)(z+u+v)>0$, since $z, v>0$ and $-z-u-v>0$. Finally, $\varepsilon_{6}>0$ since $-z / 2 v<0$ and $-w-y-x>0$, and, therefore,

$$
(u+v+z+w)^{2}<(u+v+z+w)^{2}+4 v(-w-y-x) .
$$

Then,

$$
\tilde{u}=u-\varepsilon<0 \quad \text { and } \quad \tilde{z}=z+\varepsilon>0 .
$$

Moreover, since $\varepsilon<\varepsilon_{2}=(u-x) / 2$, this assures that $\tilde{x}<\tilde{u}$, and, thus, the parameterization of $\tilde{\mathcal{M}}$ is different from that of $\mathcal{M}$ with permuted states. Next,

$$
\frac{(u-x)-\sqrt{(x-u)^{2}+4 z y}}{2}<0<\varepsilon<\frac{(u-x)+\sqrt{(x-u)^{2}+4 z y}}{2}
$$

implies that

$$
\tilde{y}(\tilde{u}, \tilde{z}) \equiv \frac{-\left(\varepsilon^{2}+(x-u) \varepsilon-z y\right)}{z+\varepsilon}>0
$$

In addition,

$$
\tilde{w}(\tilde{u}, \tilde{z}) \equiv \frac{w z+v \varepsilon}{z}>0, \quad \tilde{v} \equiv \frac{v(z+\varepsilon)}{z}>0,
$$

and, since $\varepsilon<z(1-\phi) / \phi$,

$$
\tilde{\phi} \equiv \frac{(z+\varepsilon) \phi}{z} \in[0,1] .
$$

It remains to prove that $-\tilde{z}-\tilde{u}-\tilde{v}>0$ and $-\tilde{x}-\tilde{y}-\tilde{w}>0$. It is easy to check that

$$
-\tilde{z}-\tilde{u}-\tilde{v}=-z-u-\frac{v(z+\varepsilon)}{z},
$$

which is positive if and only if $\varepsilon<\varepsilon_{5}=-(z / v)(z+u+v)$. Finally, an easy computation shows that $-\tilde{x}-\tilde{y}-\tilde{w}>0$ is equivalent to

$$
-\varepsilon-x>\frac{-\left(\varepsilon^{2}+(x-u) \varepsilon-z y\right)}{z+\varepsilon}+\frac{w z+v \varepsilon}{z},
$$

which holds if and only if $\varepsilon \in\left(r_{1}, r_{2}\right)$, where

$$
\begin{aligned}
& r_{1}=-\frac{z}{2 v}\left[(u+v+z+w)+\sqrt{(u+v+z+w)^{2}+4 v(-w-y-x)}\right]<0, \\
& r_{2}=-\frac{z}{2 v}\left[(u+v+z+w)-\sqrt{(u+v+z+w)^{2}+4 v(-w-y-x)}\right]=\varepsilon_{6}>0 .
\end{aligned}
$$


Now, let us assume that $x=u$. Then, let $\varepsilon$ be defined as in (4.9), where in this case, $\varepsilon_{3} \equiv \sqrt{z y}$. Then,

$$
\begin{aligned}
\tilde{u} & =u-\varepsilon<0 \\
\tilde{z} & =z+\varepsilon>0, \\
\tilde{x} & =x+\varepsilon<0 \quad(\text { since } \varepsilon<-x), \\
\tilde{y} & =\frac{z y-\varepsilon^{2}}{z+\varepsilon}>0 \quad(\text { since } \varepsilon<\sqrt{z y}), \\
\tilde{w} & =\frac{w z+v \varepsilon}{v}>0, \\
\tilde{v} & =\frac{v(z+\varepsilon)}{z}>0
\end{aligned}
$$

and $\tilde{\phi} \in[0,1],-\tilde{z}-\tilde{u}-\tilde{v}>0$, and $-\tilde{x}-\tilde{y}-\tilde{w}>0$ follow from the assumptions $\varepsilon<\varepsilon_{4}$, $\varepsilon<\varepsilon_{5}$, and $\varepsilon<\varepsilon_{6}$, respectively.

\section{Appendix E. Proof of Proposition 4.4}

Let us assume that

$$
\boldsymbol{G}=\left(\begin{array}{ll}
a & b \\
c & d
\end{array}\right), \quad \boldsymbol{H}=\left(\begin{array}{ll}
\alpha & \beta \\
\gamma & \delta
\end{array}\right), \quad \text { and } \quad \boldsymbol{G} \boldsymbol{H}=\left(\begin{array}{ll}
A & B \\
C & D
\end{array}\right)
$$

Now it is straightforward to verify that if $\tilde{\boldsymbol{G}}$ and $\tilde{\boldsymbol{H}}$ are defined by (4.19)-(4.22) (with respect to the elements of $\boldsymbol{G}$ and $\boldsymbol{H}$, respectively), then

$$
\tilde{\boldsymbol{G}} \tilde{\boldsymbol{H}}=\left(\begin{array}{cc}
\frac{\phi(A-C)+\tilde{\phi} C}{\phi} & \frac{\phi \tilde{\phi}(\boldsymbol{D}+2 C-A)+\phi^{2}(A-\boldsymbol{D}+B-C)-\tilde{\phi}^{2} C}{\phi \tilde{\phi}} \\
\frac{\tilde{\phi} C}{\phi} & \frac{\phi(C+\boldsymbol{D})-\tilde{\phi} C}{\phi}
\end{array}\right) .
$$

\section{Acknowledgements}

The authors are grateful to an anonymous reviewer for his/her detailed and insightful comments on an earlier version. This work has been partially supported by the Spanish research projects 2007/04438/001, 2008/00052/001, and 2009/00147/001.

\section{References}

[1] Asmussen, S. And Koole, G. (1993). Marked point processes as limits of Markovian arrival streams. J. Appl. Prob. 30, 365-372.

[2] Ausín, M. C., Wiper, M. P. And Lillo, R. E. (2008). Bayesian prediction of the transient behaviour and busy period in short- and long-tailed GI/G/1 queueing systems. Comput. Statist. Data Anal. 52, 1615-1635.

[3] Bean, N. G. And Green, D. A. (2000). When is a MAP poisson? Math. Comput. Modelling 31, 31-46.

[4] Chakravarthy, S. R. (2001). The batch Markovian arrival process: a review and future work. In Advances in Probability Theory and Stochastic Processes, Notable Publications, NJ, pp. 21-49.

[5] Fearnhead, P. and Sherlock, C. (2006). An exact Gibbs sampler for the Markov-modulated poisson process. J. R. Statist. Soc. B 68, 767-784.

[6] GREEN, D. (1998). MAP/PH/1 departure processes. Doctoral Thesis, School of Applied Mathematics, University of Adelaide.

[7] HE, Q.-M. AND ZHANG, H. (2006). PH-invariant polytopes and Coxian representations of phase type distributions. Stoch. Models 22, 383-409. 
[8] He, Q.-M. AND ZHANG, H. (2008). An algorithm for computing minimal Coxian representations. INFORMS J. Computing 20, 179-190.

[9] He, Q.-M. And Zhang, H. (2009). Coxian representations of generalized Erlang distributions. Acta Math. Appl. Sinica 25, 489-502.

[10] Heffes, H. (1980). A class of data traffic processes-covariance function characterization and related queueing results. Bell Systems Tech. J. 59, 897-929.

[11] Heffes, H. and Lucantoni, D. (1986). A Markov-modulated characterization of packetized voice and data traffic and related statistical multiplexer performance. IEEE J. Sel. Areas Commun. 4, 856-868.

[12] Heyman, D. P. and Lucantoni, D. (2003). Modeling multiple IP traffic streams with rate limits. IEEE/ACM Trans. Networking 11, 948-958.

[13] Ito, H., Armari, S.-I. And Kobayashi, K. (1992). Identifiability of hidden Markov information sources and their minimum degrees of freedom. IEEE Trans. Inf. Theory 38, 324-333.

[14] Leroux, B. G. (1992). Maximum-likelihood estimation for hidden Markov models. Stoch. Process. Appl. 40, 127-143.

[15] Lucantoni, D. M. (1993). The BMAP/G/1 queue: a tutorial. In Performance Evaluation of Computer and Communication Systems, eds L. Donatiello and R. Nelson, Springer, Berlin, pp. 330-358.

[16] Lucantoni, D. M., Meier-Hellstern, K. S. and Neuts, M. F. (1990). A single-server queue with server vacations and a class of nonrenewal arrival processes. Adv. Appl. Prob. 22, 676-705.

[17] Neuts, M. F. (1979). A versatile Markovian point process. J. Appl. Prob. 16, 764-779.

[18] Nielsen, B. F. (2000). Modelling long-range dependent and heavy-tailed phenomena by matrix analytic methods. In Advances in Algorithmic Methods for Stochastic Models, Notable Publications, pp. 265-278.

[19] Ramaswami, V. (1980). The N/G/1 queue and its detailed analysis. Adv. Appl. Prob. 12, 222-261.

[20] Ramirez, P., Lillo, R. E. AND WiPER, M. P. (2008). On identifiability of MAP processes. Technical Report, Statistics and Econometrics Working Papers ws084613, Universidad Carlos III de Madrid.

[21] RydÉn, T. (1994). Consistent and asymptotically normal parameter estimates for hidden Markov models. Ann. Statist. 22, 1884-1895.

[22] Rydén, T. (1996). An EM algorithm for estimation in Markov-modulated Poisson processes. Comput. Statist. Data Anal. 21, 431-447.

[23] RYDÉn, T. (1996). On identifiability and order of continous-time aggregated Markov chains, Markov-modulated Poisson processes, and phase-type distributions. J. Appl. Prob. 33, 640-653.

[24] ScotT, S. L. (1999). Bayesian analysis of a two-state Markov modulated Poisson process. J. Comput. Graph. Statist. 8, 662-670.

[25] Scott, S. L. And Smyth, P. (2003). The Markov modulated Poisson process and Markov Poisson cascade with applications to web traffic modeling. In Bayesian Statistics, 7 (Tenerife, 2002), Oxford University Press, New York, pp. 671-680. 